\title{
Grain-size effect on uplift capacity of plate anchors in coarse granular soils
}

\author{
S. ATHANI*, P. KHAREL*, D. AIREY* and P. ROGNON*
}

\begin{abstract}
This letter investigates the uplift capacity of plate anchors in granular soils. Simulations based on a discrete-element method are used to measure the uplift capacity of anchors of differing widths to embedment $B / H$ and width to grain-size $B / d$ ratios. Results confirm that the uplift capacity of anchors with a large $B / d$ ratio is well described by existing models developed from continuum mechanics, with no grain-size effect. In contrast, results reveal a strong deviation from these models for anchors with relatively small $B / d$ ratios. A semi-empirical model is introduced that captures this strong grain-size effect. This model is further supported by a micro-mechanical analysis, indicating that anchor uplift capacities are not only governed by a frustum mechanism predicted by continuum mechanics but also involve the mobilisation of grains surrounding this frustum. These results and model are particularly important to rationalise uplift capacities measured in small-scale experiments, typically involving small $B / d$ ratios, and to safely upscale them to larger anchor size relevant to field applications.
\end{abstract}

KEYWORDS: anchors \& anchorages; sands

Published with permission by the ICE under the CC-BY licence http://creativecommons.org/licenses/by/4.0/

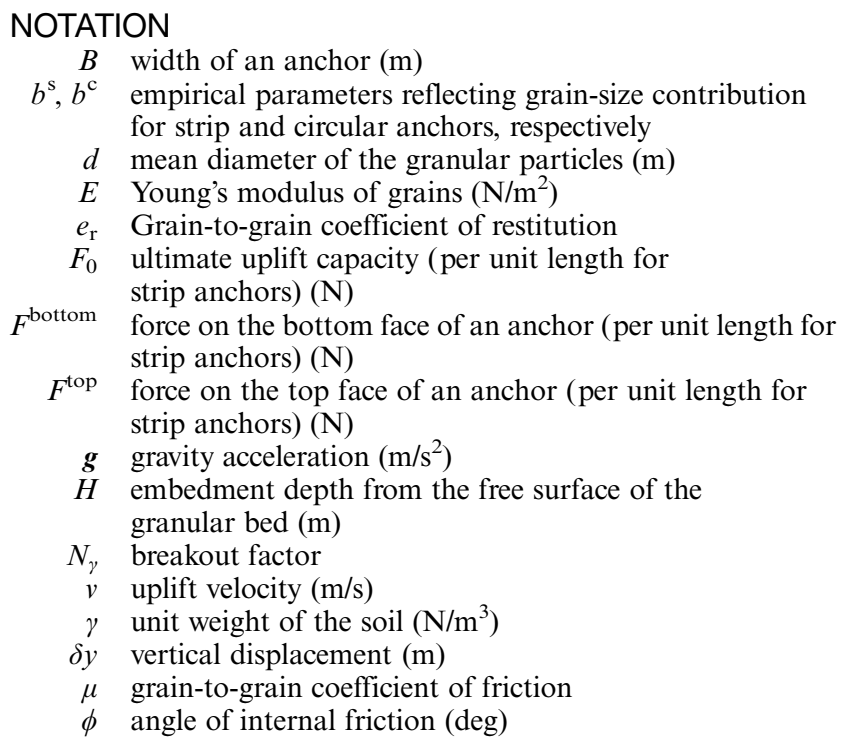

\section{INTRODUCTION}

Plate anchors are commonly used to stabilise utility poles, transmission towers and offshore infrastructures (Merifield \& Sloan, 2006; Kumar \& Kouzer, 2008; Das \& Shukla, 2013). In frictional soils like dense sands, their vertical uplift capacity $F_{0}$ is well predicted by models based on continuum mechanics (Meyerhof \& Adams, 1968; Rowe \& Davis, 1982;

Manuscript received 10 January 2017; first decision 28 March 2017; accepted 29 March 2017.

Published online at www.geotechniqueletters.com on 24 April 2017.

*School of Civil Engineering, The University of Sydney, Sydney, 2006 NSW, Australia.
Murray \& Geddes, 1987), which can be expressed in the following generic form

$$
N_{\gamma} \equiv \frac{F_{0}}{\gamma S H}=1+f\left(\frac{H}{B}\right),
$$

where $N_{\gamma}$ is a breakout factor, $\gamma$ is the soil unit weight, $S$ is the anchor surface area, $B$ is the typical anchor width and $H$ is its embedment (Fig. 1). Several expressions of the function $f$ were established to capture the measured uplift capacity of anchors of different shapes, including strip, square, rectangular, circular and fractal geometries (Meyerhof \& Adams, 1968; Murray \& Geddes, 1987; Dyson \& Rognon, 2014), embedded in soils of differing internal friction angle $\phi$. Most of these studies, however, consider large ratios of anchor-to-soil grain size, $B / d$, reflecting applications where large anchors are placed in fine sandy soils. Then, the size of the soil particles is thought to have no effect on the anchor uplift capacity.

In some applications, however, anchors are embedded in coarse granular soils such as gravels, cobbles, ballast and fragmented rocks, leading to smaller ratios $B / d$. Interestingly, with small ratios of $B / d$, studies of bearing capacities of foundation footing have detected some noticeable grain-size effects (Tatsuoka et al., 1997; Cerato \& Lutenegger, 2007). Similarly, some results suggest that uplift capacities of plate anchors would increase with the grain size for relatively small B/d (Sakai \& Tanaka, 1998; Sakai et al., 1998; Hsu \& Chang, 2007; Costantino et al., 2008).

Relatively small $B / d$ ratios are also found in most experimental set-ups dedicated to measuring uplift capacities of anchors in sandy soils. In centrifuge tests or $1 \mathrm{~g}$-chamber tests, the tested anchor size $B$ is usually between 15 and $50 \mathrm{~mm}$ (Merifield \& Sloan, 2006; Garnier et al., 2007; Chow et al., 2015; Bradshaw et al., 2016; Schiavon et al., 2016), with grain-size $d_{50}$ of few hundredths microns. This leads to $B / d$ ranging from a few dozens to a few hundreds. In practice, these results are commonly used to determine the function $f$ in equation (1), which is then 


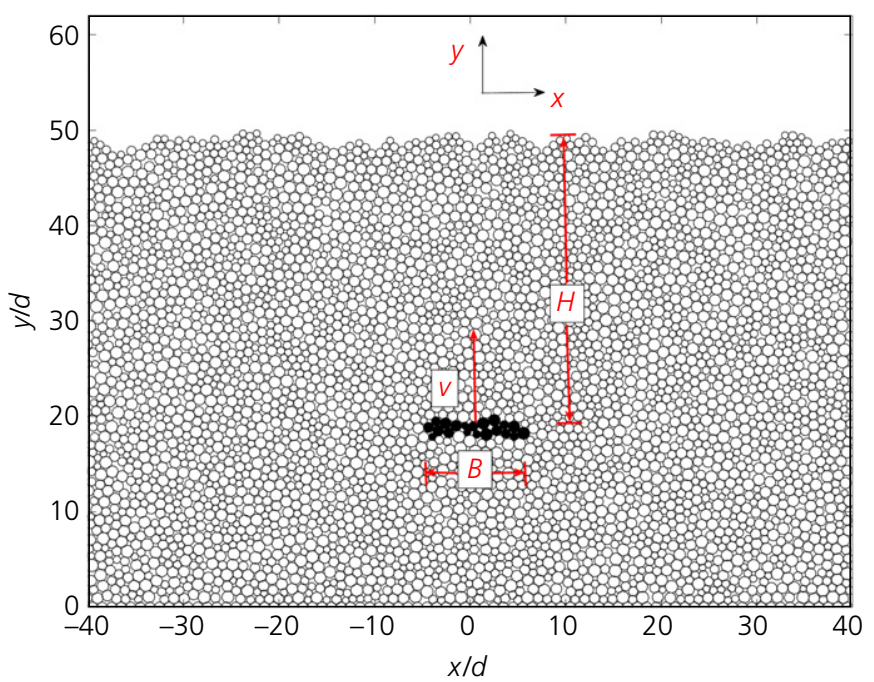

Fig. 1. Simulated system: strip anchor (black grains) embedded in a granular material (white grains). The system is periodic in the $x$-direction, and there is a layer of fixed grains at $y=0$

directly upscaled to design anchors up to a few metres wide in sand. However, such full-scale anchors are characterised by much larger $B / d$ ratios of the order of $10^{4}$. There is thus a risk that the uplift capacities measured in the laboratory could include a grain-size contribution which does not exist in real applications. Failure to account for this grain-size contribution when upscaling could therefore lead to an overestimation of the full size anchor uplift capacity, and to unsafe designs. However, grain-size effects are yet to be fully quantified. Specifically, there is no established model such as equation (1) that would account for a grain-size contribution and allows for a safer upscaling of plate anchor uplift capacity.

The purpose of this letter is to identify and rationalise the grain-size effect on the uplift capacity of plate anchors. In this aim, the uplift capacities of anchors of differing embedment ratios $H / B$ and width ratios $B / d$ are measured using a discrete-element method (DEM). These data will then serve as a benchmark to identify how a model such as equation (1) could be extended to capture grain-size effects and to identify the micro-mechanisms governing these effects.

\section{MEASURING UPLIFT CAPACITY USING DEM}

The uplift of strip anchors is simulated using a DEM in a two-dimensional periodic system, as illustrated in Fig. 1. The dimension of the test chamber is $8 B$ in the $x$-direction, and the anchors are placed at a distance $2 B$ from the bottom. The authors systematically checked that further increasing the width of the chamber, or the distance to the bottom, does not affect the uplift capacities.

The granular material under consideration is comprised of several thousand disks of mean diameter $d \equiv d_{50}$ and mass $m$. A polydispersity of $d \pm 30 \%$ is introduced to avoid grain crystallisation. Grains interact with their neighbours by way of inelastic and frictional contacts characterised by Young's modulus $E=1000\left(\mathrm{mg} / \mathrm{d}^{2}\right)$, coefficient of restitution $e_{\mathrm{r}}=0 \cdot 5$ and coefficient of friction $\mu=0 \cdot 5$. Grains are initially placed loosely without contact. They then settle under the action of gravity $g$, until the total kinetic energy of the system becomes negligible. This creates a homogeneous and densely packed configuration (solid fraction of $\sim 0 \cdot 8$ ). The critical state internal friction angle of such configurations was measured using a plane shear test and found to be approximately $\phi \approx 15 \cdot 5^{\circ}$, which is typical of two-dimensional packings of frictional disks (da Cruz et al., 2005; Voivret et al., 2009).

Anchors of width $B$ and thickness $2 d$ are defined in these dense configurations by selecting grains at a desired location, as shown in Fig. 1. This method ensures that the anchor placement does not disturb the microstructure of the material. The anchor grains are then moved according to a prescribed uplift velocity selected to be $v=0 \cdot 1 \sqrt{\mathbf{g} d}$. Slower velocities were also tested, with no noticeable effect on the uplift capacities. Individual grain motions are integrated over small time step $d t$ using a predictor-corrector numerical scheme. More details about the numerical scheme and the contact law can be found in Rognon et al. (2015).

Figure 2(a) shows the variation of the total force $F$ per unit length experienced by an anchor (excluding its weight) as a function of its vertical displacement $\delta y$, relative to its initial position. The anchor width is $B=10 d$, and results with different embedment ratios are shown. They indicate a sharp

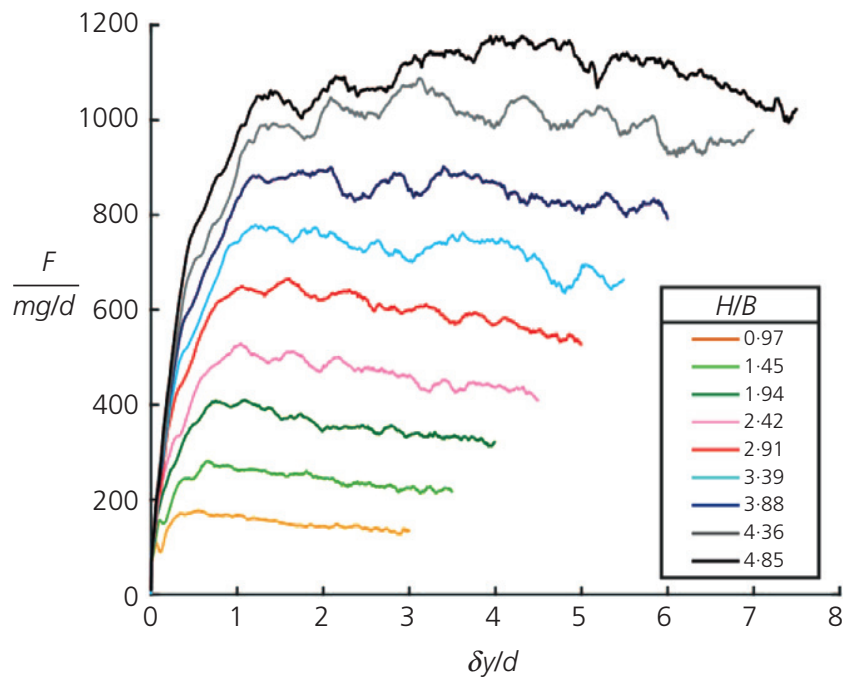

(a)

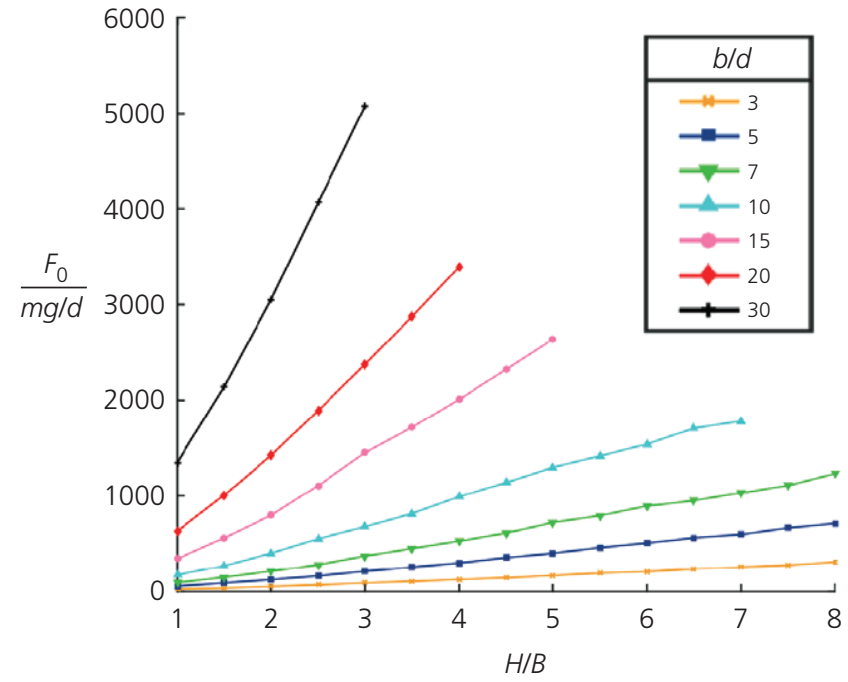

(b)

Fig. 2. Simulated uplift forces for strip anchors. (a) Force-displacement during the pullout for a strip anchor of width $10 d$ and different embedments - $\delta y$ represents the vertical displacement of the anchor, and $F$ represents the total force it experiences. (b) Uplift capacities $F_{0}$ for different anchor sizes and different embedment ratios 
increase in force for small displacements followed by a slow decrease in force, which is consistent with existing anchor force-displacement measurements (Rowe \& Davis, 1982; Dickin, 1988). The anchor uplift capacity $F_{0}$ is defined as the maximum of $F(\delta y)$ for each test. The fluctuations in forces observed in Fig. 2(a) result from the rearrangements of grains induced by the anchor motion. Each test was repeated ten times with different realisation of the initial packings to obtain the corresponding average uplift capacity $F$. Figure 2(b) shows the uplift capacities $F_{0}$ thus obtained for anchors of differing widths in the range $3 \leq B / d \leq 30$ and different embedment ratios in the range $1 \leq H / B \leq 8$ (For instance, in gravels of size $d \approx 10 \mathrm{~mm}$, an embedment ratio $H / B=3$ and a width ratio $B / d=30$ correspond to an anchor of width $B=300 \mathrm{~mm}$ at a depth $H=900 \mathrm{~mm}$; in cobbles, ballast or fragmented rocks of grain-size $d \approx 100 \mathrm{~mm}$, an embedment ratio $H / B=1$ and a width ratio $B / d=5$ correspond to an anchor of width $B=500 \mathrm{~mm}$ at a depth $H=500 \mathrm{~mm}$.). These results indicate that both ratios strongly influence the uplift capacity.

Figure 3 highlights the effect of the grain-size $d$ on the breakout factor $N_{\gamma}$, as defined by equation (1). It shows that, for a given embedment ratio, two anchors of different width ratios may present significantly different breakout factors. Breakout factors of the smallest anchors $(B=3 d)$ are $\sim 75 \%$ larger than those of the largest tested anchors $(B=30 d)$.

\section{MODELLING GRAIN-SIZE EFFECT ON UPLIFT CAPACITY OF STRIP ANCHORS}

The breakout factor of strip anchor in a Mohr-Coulomb soil is usually modelled by equation (1) using a function $f^{\mathrm{s}} \approx \tan \phi(\mathrm{H} / B)$ (Meyerhof \& Adams, 1968; Murray \& Geddes, 1987). This leads to the following breakout formula:

$$
N_{\gamma}^{\mathrm{s}}=1+\tan \phi \frac{H}{B}
$$

which does not include any grain-size contribution. Data shown in Fig. 3 indicate that the breakout factor increases almost linearly with $d / B$, for every embedment ratio $H / B$. Accordingly, the authors introduce the following extension of the model in equation (2) to account for the grain size

$$
N_{\gamma}^{\text {strip }}=N_{\gamma}^{\mathrm{s}}(H / B)+b^{\mathrm{s}} \frac{d}{B} .
$$

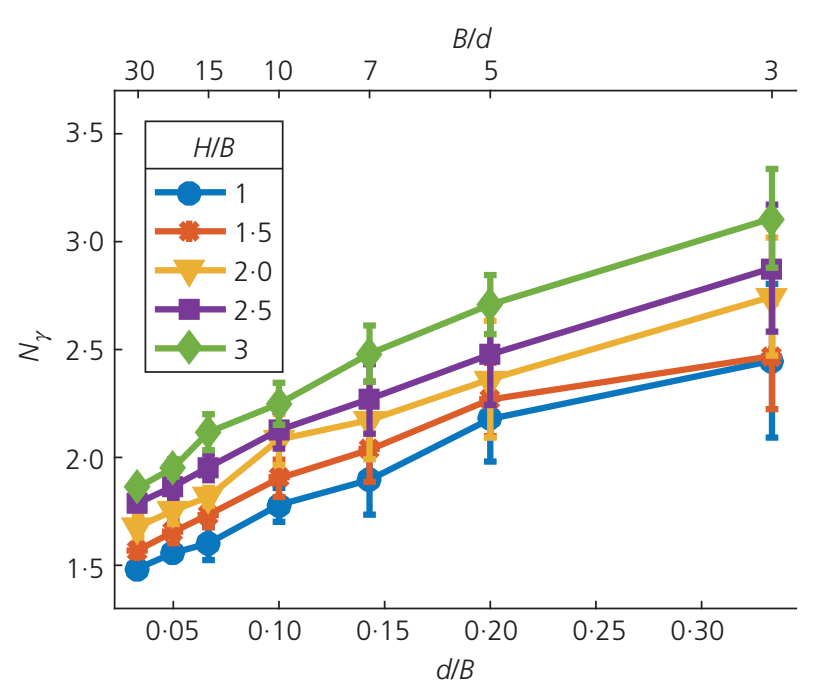

Fig. 3. Simulated breakout factor $N_{\gamma}$ for strip anchors of differing width and embedment ratios. The error bars denote the standard deviation of the measured breakout factor considering ten tests (see text) where $b^{\mathrm{s}}$ is an empirical parameter reflecting the grain-size contribution. Figure 4 compares the measured breakout factors with the two models in equations (2) and (3) using $\phi=15 \cdot 5^{\circ}$ and $b_{\mathrm{s}}$ as a free parameter. It appears that the predicted breakout factors ignoring the grain-size effect consistently underestimate the actual breakout factor. In contrast, it is found that the proposed model (3) captures all the measured breakout factors using a single value of $b^{\mathrm{s}}=4 \cdot 5$.

Interestingly, Fig. 4 also reveals that the breakout factors tend to plateau for large embedment ratios $H / B$. In the context of large anchors, this effect is known and corresponds to a transition between 'shallow' anchors, for which equation (2) is valid, and 'deep' anchors characterised by a constant breakout factor. For strip anchors, this transition occurs for embedment ratios larger than $\sim 2.5$ (Meyerhof \& Adams, 1968). Results shown in Fig. 4 suggest that this transition occurs for larger embedment ratios, up to 5 , for anchors with smaller $B / d$ ratios. This indicates that the grain size not only affects the behaviour of shallow anchors but also affects the transition towards deep anchor conditions and the corresponding ultimate breakout factor.

\section{MODELLING GRAIN-SIZE EFFECT ON UPLIFT CAPACITY OF CIRCULAR ANCHORS}

The breakout factor of shallow circular anchor differs from that of strip anchors due to their difference in shape. It is usually modelled by a function $f^{\mathrm{c}}=(1 / 3)[(1+2 \tan \phi$ $\left.(\mathrm{H} / d))^{2}+2 \tan \phi(\mathrm{H} / d)-1\right]$, leading to the following expression for the breakout factor (Meyerhof \& Adams, 1968; Dyson \& Rognon, 2014)

$$
N_{\gamma}^{\mathrm{c}}=\frac{1}{3}\left[\left(1+2 \tan \phi \frac{H}{d}\right)^{2}+2 \tan \phi \frac{H}{d}+2\right],
$$

which does not involve any grain-size effect. Following the modelling approach developed for strip anchors in equation (3), the authors propose to model the grain-size effect on circular anchors breakout factors by adding a term proportional to $d / B$ :

$$
N_{\gamma}^{\text {circular }}=N_{\gamma}^{\mathrm{c}}(H / B)+b^{\mathrm{c}} \frac{d}{B} .
$$

The uplift capacity of circular anchors in dense sand has been experimentally measured by Sakai \& Tanaka (1998) and Sakai et al. (1998), covering a range of embedment ratio $1 \leq H / B \leq 3$ and anchor width ratio $7 \cdot 10^{-4} \leq d / B \leq 5 \cdot 10^{-3}$ provides an opportunity to assess the proposed model. Figure 5 compares these results to the prediction of equations (4) and (5), using $\phi=35^{\circ}$ and $b^{\mathrm{c}}$ as a free parameter. It appears that, for each embedment ratio, the breakout factor increases approximately linearly with $d / B$, confirming the validity of the proposed model in equation (5). Furthermore, it is found that all measured breakout factors for different $H / B$ and $d / B$ ratios are captured by using a single value of $b^{\mathrm{c}} \approx 240$.

\section{MICRO-MECHANISMS LEADING TO GRAIN-SIZE EFFECT}

The continuum models in equations (2) and (4) are based on a simple 'frustum' mechanism: they both consider that the upward anchor motion mobilises a frustum of soil located above it and equate the anchor uplift capacity to the weight of this frustum. Frustums are delineated by failure planes originating from the anchor edges, and propagating upward 


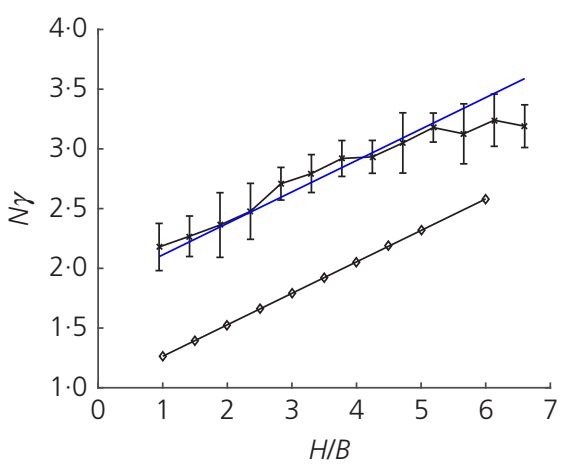

(a)

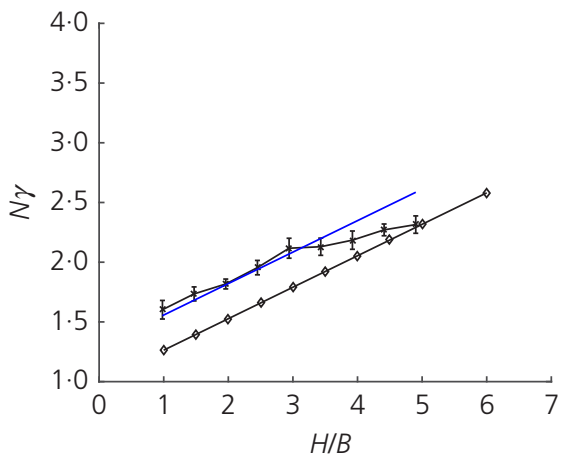

(d)

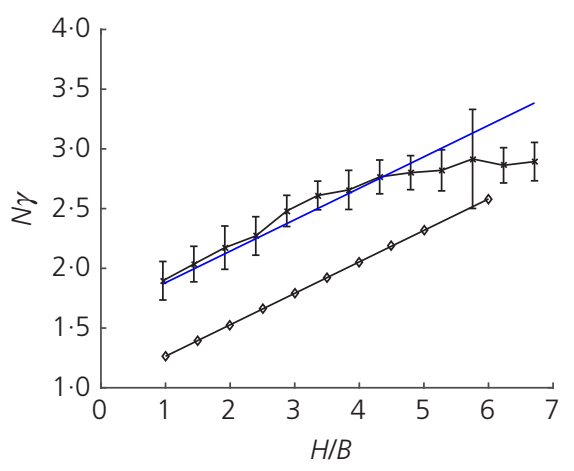

(b)

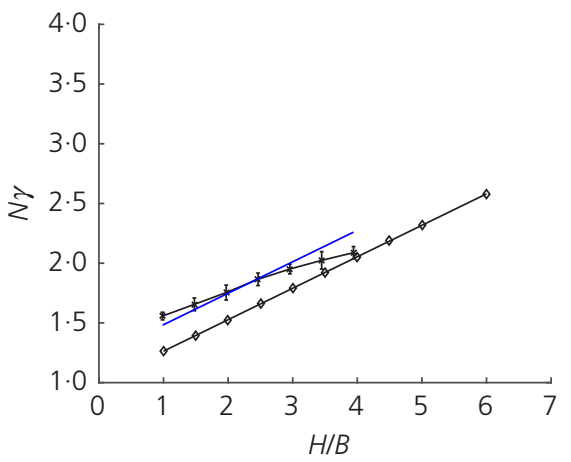

(e)

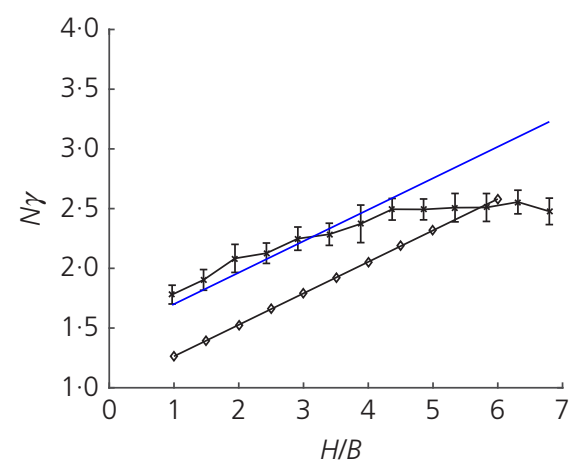

(c)

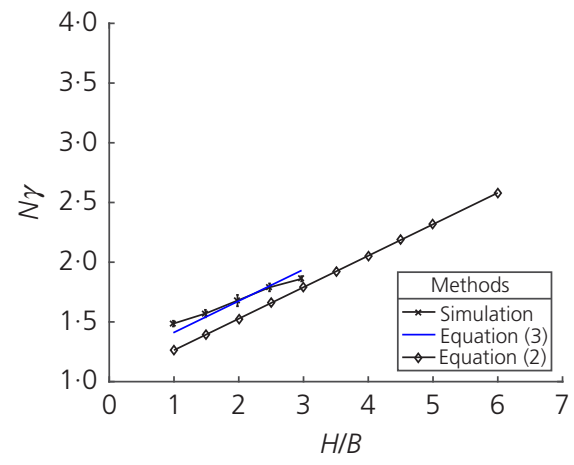

(f)

Fig. 4. Simulated breakout factor for strip anchors of differing width and embedment ratios (cross markers) compared to the predictions of the model in equation (2) which does not include a grain-size contribution (black line with diamond markers), and to the prediction of the model in equation (3) including some grain-size contribution (blue line without marker). (a) $B=5 d$, (b) $B=7 d$, (c) $B=10 d$, (d) $B=15 d$, (e) $B=20 d$ and (f) $B=30 d$. Predictions of equations (2) and (3) are obtained using $\phi=15 \cdot 5^{\circ}$ and $b^{\mathrm{s}}=4 \cdot 5$

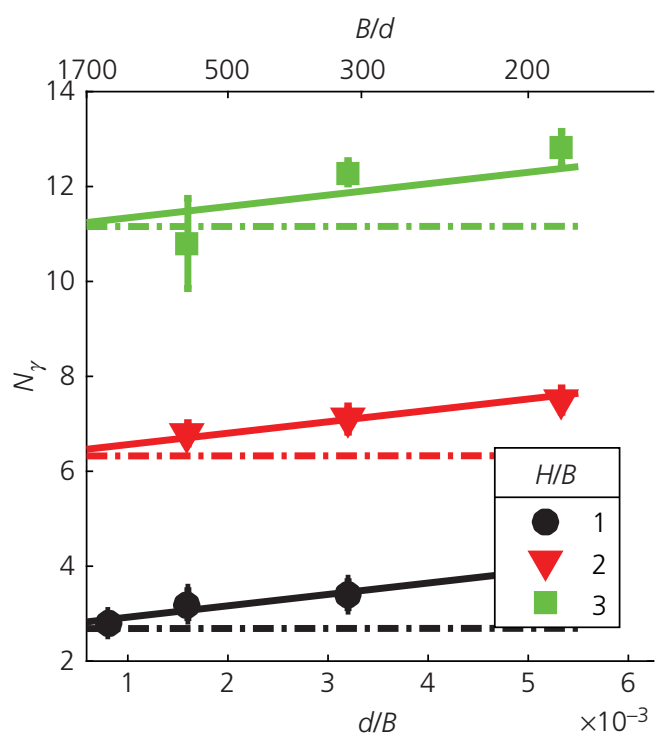

Fig. 5. Breakout factors for circular anchors in sand for different embedment and width ratios: experimental data from Sakai \& Tanaka (1998) and Sakai et al. (1998) (markers), prediction of continuum-based model in equation (4) using $\phi=35^{\circ}$ (dashed line) and prediction of the proposed model in equation (5), including a grain-size contribution (continuous line)

with an inclination $\phi$ relative to the vertical (different inclinations are sometimes considered to match the experimental data). According to this picture, the frustum is a truncated pyramid for a strip anchor and a truncated cone for a circular anchor.
This mechanism, however, is independent of the grain size. This section now focuses on analysing micro-structural information available from DEM simulations to identify (a) whether such frustum can be observed and $(b)$ what other micro-mechanisms could lead to a grain-size contribution. In this aim, Figs 6 and 7 compare the internal grain displacement and force distribution for two strip anchors of similar embedment $H / B=1$ but different width ratios, $B / d=10$ and 30 .

Figures 6(a) and 7(a) represent all the contact forces between grains in the initial configurations, before the anchor is moved. On these figures, each contact between two grains is represented by a red line joining the two grain centres. Line widths are proportional to the magnitude of the normal contact forces, which are purely compressive as there is no adhesive force between grains. The figures evidence the expected increase in contact compression with depth, reflecting the increase in normal stresses due to gravity. Furthermore, this representation highlights a large distribution of contact compressive forces. The contact network exhibits chains of highly compressed grains while other contacts are much less compressed, which is typical of granular packings (Radjai et al., 1998; Majmudar \& Behringer, 2005).

Figures 6(a) and 7(a) also show that before the anchor is moved, it is subjected to compressive forces on both its top and bottom surfaces. The anchor is thus subjected to a total vertical force $F^{\text {top }}$ due to compressive contacts acting on the top, which pushes it down and resists its upward motion. It is simultaneously subjected to a total vertical force $F^{\text {bottom }}$ due to compressive contacts acting on its bottom pushing it up. At equilibrium, these two forces balance the anchor weight.

Figures 6(b) and 7(b) show the evolution of the two forces $F^{\text {top }}$ and $F^{\text {bottom }}$, and of the total pullout force 


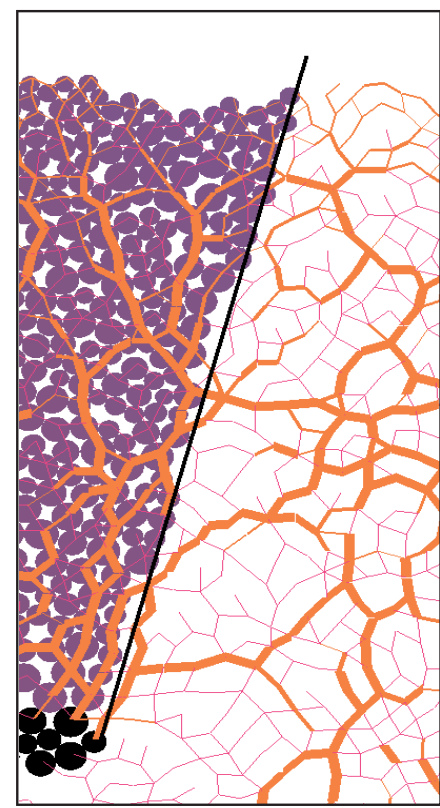

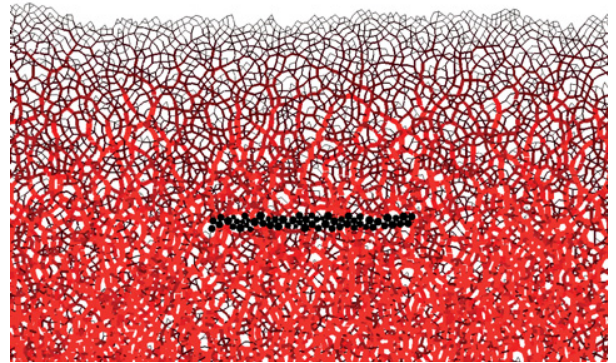

(a)

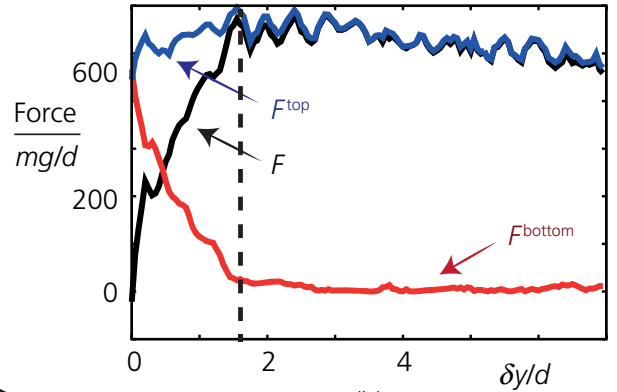

(b)
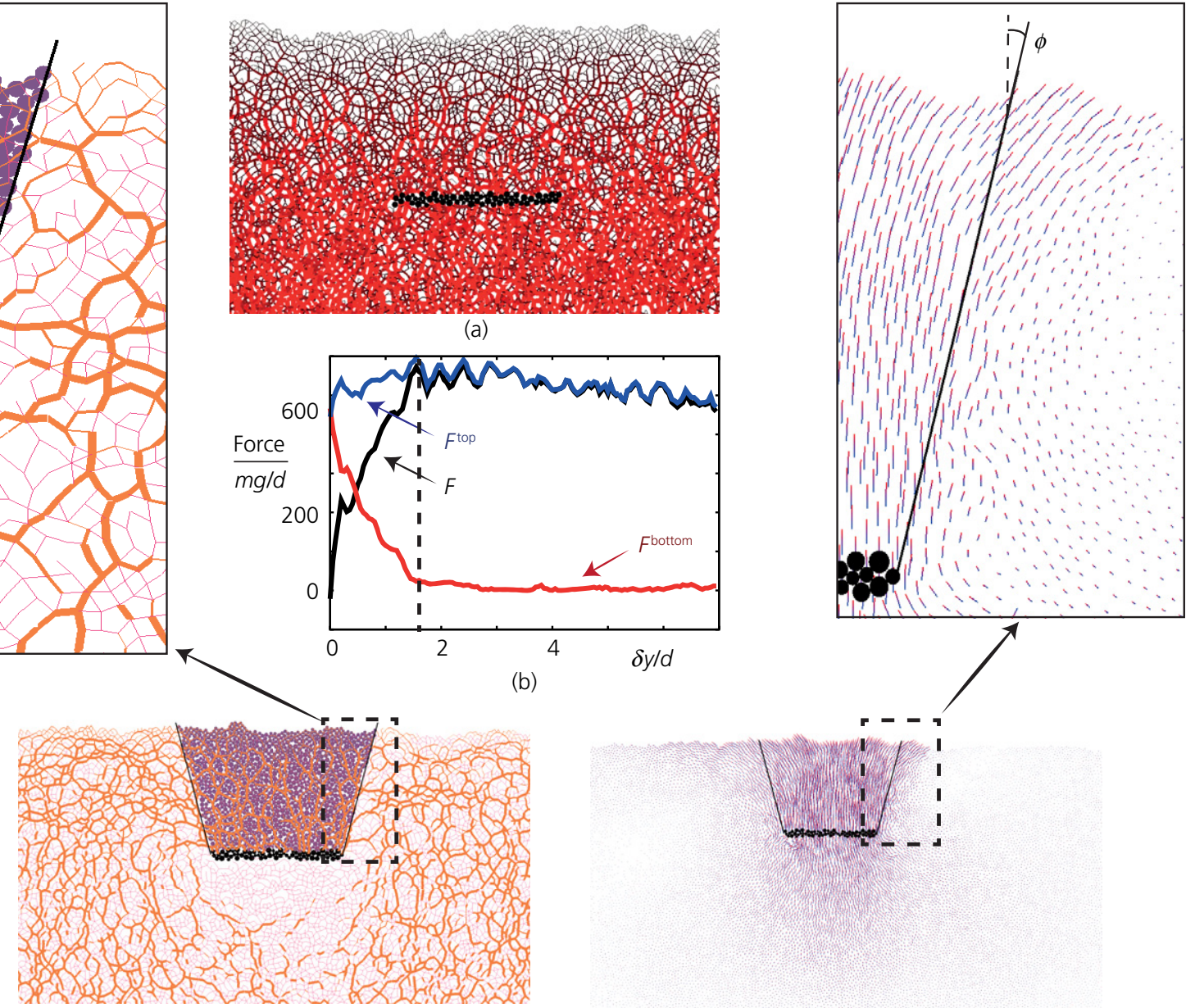

(c)

(d)

Fig. 6. Microstructure evolution during the anchor uplift for an anchor with an embedment ratio $H / B=1$ and a width ratio $B / d=30$. (a) Contact network before anchor motion (see text). (b) Total force $F$, force on the top face $F^{\text {top }}$ and force on the bottom face $F^{\text {bottom }}$ of the anchor during uplift, as a function of the anchor vertical displacement $\delta y$. (c) Contact network at failure (see text). (d) Displacement field of individual grains; each line connects the initial position (blue) of a grain to its position when failure occurs (red). In (c) and (d), the black lines represent the frustum boundary, with an inclination of $15.5^{\circ}$ from vertical. Note that (a), (c), (d) only represent a small part of the simulated test chamber

$F=F^{\text {top }}-F^{\text {bottom }}$ while the anchor is moving upward. In both cases, the compressive force at the top first increases while the compressive force at the bottom decreases. This leads to a sharp increase in total force. Once $F^{\text {bottom }}$ reaches zero, denoting a complete disconnection between the bottom of the anchor and the grains, $F^{\text {top }}$ starts decreasing leading to a decrease in the total force. As a consequence, the failure is found to coincide with the disconnection of the bottom part of the anchor from the grains. At failure, the pullout force is thus entirely driven by the weight of the grains located above the anchor, which is qualitatively consistent with the frustum mechanism.

Figures 6(c) and 7(c) illustrate the mode of force transmission near the anchor at failure by showing the contact network. On these figures, the orange lines represent contacts. Their width is proportional to the magnitude of the contact force minus the typical average contact force at the contact position $y_{\mathrm{c}}, \gamma d^{2}\left(H-y_{\mathrm{c}}\right)$. This highlights the existence of highly compressed contact chains across the depth. These figures also represent the grains within the theoretical frustum. Thus, they show that long chains of highly compressed contacts develop not only vertically above the anchor within the frustum but also sideways, originating from the anchor edge and crossing the frustum boundary.
Figures 6(d) and 7(d) show the effect of these force chains on the grain displacements at failure. It appears that grains located within the frustum are mostly moving upward along with the anchor, which is consistent with the frustum mechanism. However, grains surrounding the frustum also appear to be mobilised. This mobilisation of the grains within a skirt around the frustum, which can be attributed to some shear stress at the frustum interface, is not accounted for by the frustum mechanism and is expected to lead to an additional contribution to the anchor uplift capacity.

Accounting for the weight of a skirt of width $b d$ in the pullout capacity would lead to add a breakout factor contribution proportional to $b d / B$, which is consistent with the measurements and proposed models equations (3) and (5). This suggests that the grain-size effect originates from this skirt mechanism. Considering that the weight of the skirt is proportional to the frustum surface area and thus to the anchor size $B$, while the frustum weight is proportional to its volume and to $B^{2}$ supports the observation that grains size effects are large for small anchors, but becomes negligible for large anchors.

To date, there is no established model to quantitatively predict the width of the skirt as a function of the soil internal friction angle and/or other parameters. As a result, the parameters $b^{\mathrm{s,c}}$ cannot be predicted for a particular type 


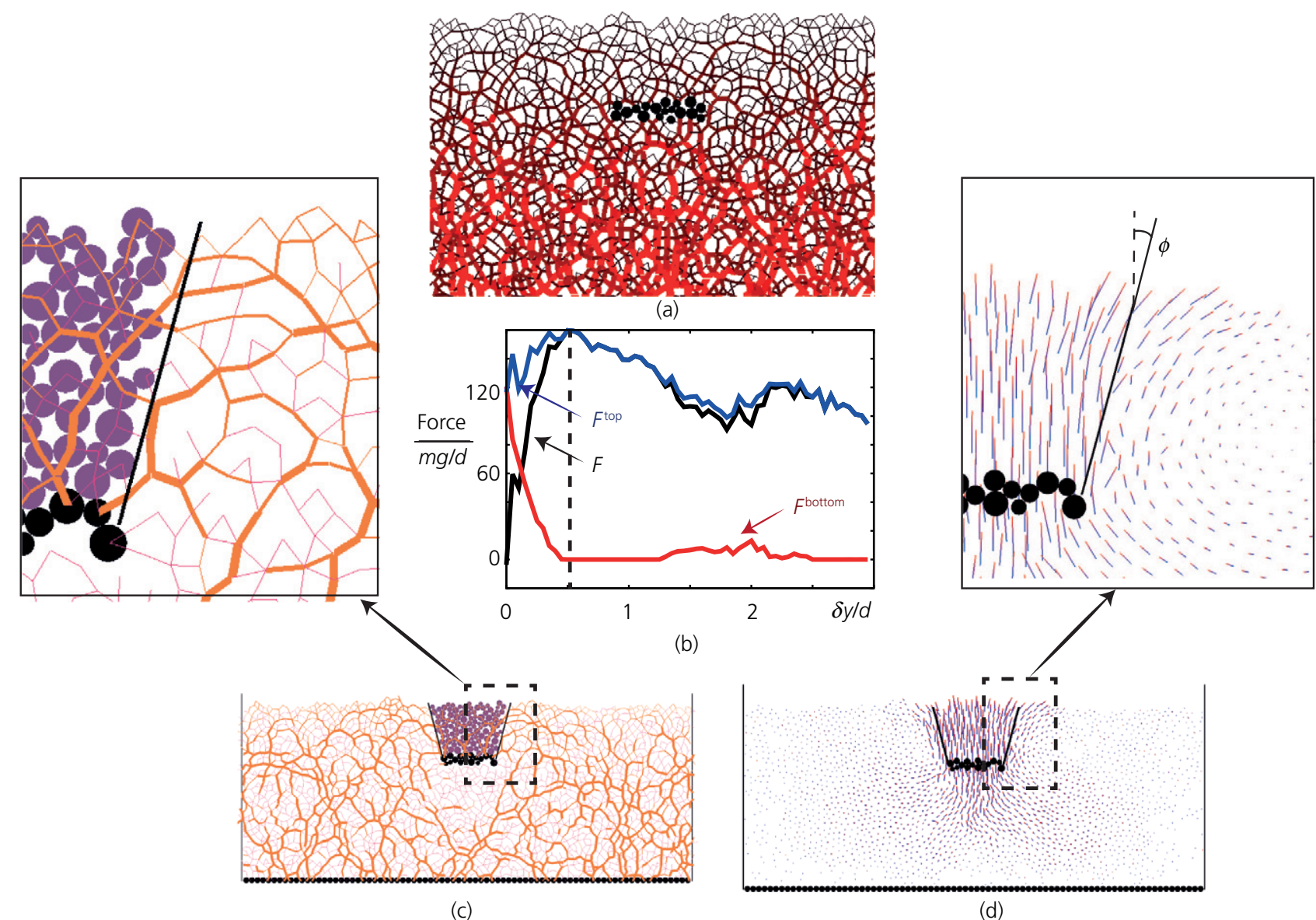

Fig. 7. Microstructure evolution during the anchor uplift for an anchor with an embedment ratio $H / B=1$ and a width ratio $B / d=10$ (see Fig. 6 caption)

of soil. Nonetheless, they can be back-analysed by fitting model (3) or (5) to experimental uplift measurements.

\section{CONCLUSION}

The results presented in this letter indicate that the uplift capacity of anchors in granular soils depends on both the macroscopic strength parameters, such as internal friction angle, and the anchor to grains size ratio. Both DEM results and experimental results from Sakai \& Tanaka (1998) and Sakai et al. (1998) suggest that the grain-size contribution on the anchor breakout factor is linearly increasing with the ratio $d / B$. Formulae (3) and (5) were introduced accordingly to capture this effect.

These results and models can be used to predict the pullout capacity of relatively small anchors in coarse gravels, pebbles and ballast, where the ratio $B / d$ is small and the grain-size contribution is significant. They also confirm that the grain-size contribution is negligible for large anchors in sandy soils, characterised by large $B / d$ ratios. In contrast, they indicate that grain-size effect can significantly contribute to the pullout capacity measured in small-scale laboratory testing, where anchors size are often limited to few centimetres.

This points out a significant risk of unsafe design when upscaling small-scale laboratory measurements to meter-size anchors used in practice. If the upscaling is performed using a formula such as equation (1) ignoring the grain-size effect, it could possibly significantly overestimate the pullout capacity of the full-scale anchor. The proposed model (5) can readily be used to estimate the magnitude of the grain-size effect in laboratory experiments and to safely upscale these small-scale measurements allowing for the grain-size contribution.

\section{REFERENCES}

Bradshaw, A., Giampa, J., Gerkus, H., Jalilvand, S., Fanning, J., Nanda, S., Gilbert, R., Gavin, K. \& Sivakumar, V. (2016). Scaling considerations for 1 -g model horizontal plate anchor tests in sand. Geotech. Test. J. 39, No. 6, 1006-1014.

Cerato, A. B. \& Lutenegger, A. J. (2007). Scale effects of shallow foundation bearing capacity on granular material. J. Geotech. Geoenviron. Engng 133, No. 10, 1192-1202.

Chow, S. H., O'Loughlin, C. D., Corti, R., Gaudin, C. \& Diambra, A. (2015). Drained cyclic capacity of plate anchors in dense sand: experimental and theoretical observations. Géotech. Lett. 5, No. 2, 80-85, http://dx.doi.org/10.1680/ geolett.15.00019

Costantino, D. J., Scheidemantel, T. J., Stone, M. B., Conger, C., Klein, K., Lohr, M., Modig, Z. \& Schiffer, P. (2008). Starting to move through a granular medium. Phys. Rev. Lett. 101, No. 10 , 108001-1-108001-4.

da Cruz, F., Emam, S., Prochnow, M., Roux, J. N. \& Chevoir, F. (2005). Rheophysics of dense granular materials: discrete simulation of plane shear flows. Phys. Rev. E 72, No. 2, 021309.

Das, B. M. \& Shukla, S. K. (2013). Earth anchors. J. Ross Publishing, Plantation, FL, USA.

Dickin, E. A. (1988). Uplift behavior of horizontal anchor plates in sand. J. Geotech. Engng 114, No. 11, 1300-1317.

Dyson, A. S. \& Rognon, P. G. (2014). Pull-out capacity of tree root inspired anchors in shallow granular soils. Géotech. Lett. 4 No. 4, 301-305, http://dx.doi.org/10.1680/geolett.14.00061.

Garnier, J., Gaudin, C., Springman, S., Culligan, P., Goodings, D., Konig, D., Kutter, B., Phillips, R., Randolph, M. \& Thorel, L. (2007). Catalogue of scaling laws and similitude questions in geotechnical centrifuge modelling. Int. J. Phys. Model. Geotech. 7, No. 3, 1, http://dx.doi.org/10.1680/ijpmg.2007.070301. 
Hsu, S. C. \& Chang, C. M. (2007). Pullout performance of vertical anchors in gravel formation. Engng. Geol. 90, No. 1-2, 17-29.

Kumar, J. \& Kouzer, K. M. (2008). Vertical uplift capacity of horizontal anchors using upper bound limit analysis and finite elements. Can. Geotech. J. 45, No. 5, 698-704.

Majmudar, T. S. \& Behringer, R. P. (2005). Contact force measurements and stress-induced anisotropy in granular materials. Nature 435, No. 7045, 1079-1082.

Merifield, R. S. \& Sloan, S. W. (2006). The ultimate pullout capacity of anchors in frictional soils. Can. Geotech. J. 43, No. 8, 852-868.

Meyerhof, G. G. \& Adams, J. I. (1968). The ultimate uplift capacity of foundations. Can. Geotech. J. 5, No. 4, 225-244.

Murray, E. J. \& Geddes, J. D. (1987). Uplift of anchor plates in sand. J. Geotech. Engng 113, No. 3, 202-215.

Radjai, F., Wolf, D. E., Jean, M. \& Moreau, J. J. (1998). Bimodal character of stress transmission in granular packings. Phys. Rev. Lett. 80, No. 1, 61.

Rognon, P. G., Miller, T., Metzger, B. \& Einav, I. (2015). Long-range wall perturbations in dense granular flows. J. Fluid Mech. 764, 171 .
Rowe, R. K. \& Davis, E. H. (1982). Behaviour of anchor plates in sand. Géotechnique 32, No. 1, 25-41, http://dx.doi.org/10.1680/ geot.1982.32.1.25.

Sakai, T. \& Tanaka, T. (1998). Scale effect of a shallow circular anchor in dense sand. Soils Found. 38, No. 2, 93-99.

Sakai, T., Erizal, V. \& Tanaka, T. (1998). Particle size effect of anchor problem with granular materials. In Application of numerical methods to geotechnical problems, (ed. A. Cividini), vol. 397, pp. 191-200. Vienna, Austria: Springer.

Schiavon, J. A., Tsuha, C. d. H. C. \& Thorel, L. (2016). Scale effect in centrifuge tests of helical anchors in sand. Int. J. Phys. Model. Geotech. 16, No. 4, 185-196, http://dx.doi.org/10.1680/jphmg. 15.00047 .

Tatsuoka, F., Goto, S., Tanaka, T., Tani, K. \& Kimura, Y. (1997). Particle size effects on bearing capacity of footing on granular material. Proceedings of international symposium on deformation and progressive failure in geomechanics (IS-Nagoya '97), pp. 133-138. Oxford, UK: Pergamon.

Voivret, C., Radjai, F., Delenne, J. Y. \& El Youssoufi, M. S. (2009). Multiscale force networks in highly polydisperse granular media. Phys. Rev. Lett. 102, No. 17, 178001.

\section{HOW CAN YOU CONTRIBUTE?}

To discuss this paper, please submit up to 500 words to the editor at journals@ice.org.uk. Your contribution will be forwarded to the author(s) for a reply and, if considered appropriate by the editorial board, it will be published as a discussion in a future issue of the journal. 